'Escuela de Medicina, Facultad de Medicina, Pontificia Universidad Católica de Chile. Santiago, Chile.

${ }^{2}$ Departamento de Medicina Interna, Facultad de Medicina, Pontificia Universidad Católica de Chile. Santiago, Chile. ${ }^{3}$ Departamento de

Endocrinología, Facultad de Medicina, Pontificia Universidad Católica de Chile. Santiago, Chile.

${ }^{4}$ Centro Traslacional de

Endocrinología (CETREN UC), Facultad de Medicina, Pontificia

Universidad Católica de Chile. Santiago, Chile. ${ }^{5}$ Departamento de HematologíaOncología, Facultad de Medicina, Pontificia Universidad Católica de Chile. Santiago, Chile.

${ }^{6}$ Sección de Nefrología, Hospital Clínico Universidad de Chile. Santiago, Chile.

${ }^{7}$ Centro de Investigación Clínica Avanzada, Hospital Clínico Universidad de Chile. Santiago, Chile.

Trabajo no recibió financiamiento. Los autores declaran no tener conflictos de interés.

Recibido el 16 de diciembre de 2019, aceptado el 26 de marzo de 2020.

Correspondencia a: Dr. Pablo Florenzano V. Departamento de Endocrinología, Escuela de Medicina, Pontificia Universidad Católica de Chile. Diagonal Paraguay 362, Cuarto piso. Santiago, Chile. pflorenz@uc.cl

\section{Hipofosfatemia inducida por drogas: fierro carboximaltosa e imatinib. Casos clínicos}

\author{
DANISA IVANOVIC-ZUVIC ${ }^{1,2}$, ALEXANDRA FISCHMAN ${ }^{1}$, \\ MACARENA JIMÉNEZ ${ }^{1}$, ALEJANDRA MARTÍNEZ ${ }^{1,3,4}$, \\ DANIEL ERNST ${ }^{1,5}$, LUIS TORO ${ }^{6,7}$, \\ FRANCISCO J. GUARDA ${ }^{1,3,4}$, PABLO FLORENZANO ${ }^{1,3,4}$
}

\section{Hypophosphatemia induced by carboxymaltose iron and imatinib. Report of two cases}

Hypophosphatemia is a relatively frequent and a potentially serious adverse drug effect. Clinically it is characterized by bone pain and muscle weakness. There are several mechanisms by which a drug can induce hypophosphatemia and they can be classified according to whether or not they are mediated by an excess of Fibroblast Growth Factor 23 (FGF23). We report two patients with the condition: (i) A 49-year-old woman with Chronic Myeloid Leukemia $(C M L)$ and gastric sleeve surgery at 46 years of age. After receiving intravenous carboxymaltose iron in one occasion due to refractory anemia, she developed symptomatic hypophosphatemia. Urinary phosphate losses associated with high FGF23 levels were confirmed. Plasma phosphate returned to normal values 90 days after the iron administration. (ii) A 40-year-old man with a history of CML in whom imatinib was started. He developed symptomatic hypophosphatemia due to non FGF23-mediated hyperphosphaturia. As treatment with imatinib could not be interrupted, hypophosphatemia and its symptoms resolved with oral phosphate intake. These cases illustrate the importance of recognizing and treating drug-induced hypophosphatemia in a timely manner, and thus avoid the morbidity associated with this entity.

(Rev Med Chile 2020; 148: 404-408)

Key words: Drug-Related Side Effects and Adverse Reactions; Fibroblast Growth Factors; Iron Compounds; Hypophosphatemia; Imatinib Mesylate.
L a hipofosfatemia se define como la disminución en la concentración de fosfato sérico en adultos con valores menores a 2,6 mg/dL ${ }^{1}$. Su prevalencia varía ampliamente según la población estudiada, siendo descrito en $5 \%$ de los pacientes hospitalizados y en $30 \%$ a $50 \%$ en pacientes alcohólicos, con sepsis severa o politraumatizados ${ }^{2}$.

Las manifestaciones clínicas dependerán del tiempo de instalación, así como del grado de hipofosfatemia. Es así como esta condición puede tener manifestaciones clínicas graves, derivadas de la disminución en la síntesis de ATP a nivel cardiopulmonar, muscular, hematológico, óseo o del sistema nervioso central, especialmente con niveles inferiores a $1 \mathrm{mg} / \mathrm{dL}^{1}$.

La regulación del fosfato está determinada por su absorción en el tracto gastrointestinal, las pérdidas urinarias y su depósito o resorción en el hueso. Estos mecanismos son regulados por la hormona paratiroidea (PTH), el factor de cre- 
cimiento de fibroblastos 23 (FGF23) y vitamina D. FGF23 se ha identificado como la principal hormona fosfatúrica al disminuir la reabsorción renal de fósforo a nivel tubular por medio de la internalización de los transportadores de sodio y fósforo en la membrana apical de las células del túbulo contorneado proximal ${ }^{3}$.

Las causas de hipofosfatemia pueden clasificarse según su mecanismo fisiopatológico en aquellas mediadas por FGF23 y aquellas que son FGF23 independientes ${ }^{3}$.

Presentaremos dos casos clínicos que ejemplifican cada una de estos grupos, destacando su importancia al ser un efecto adverso poco conocido de drogas que son ampliamente utilizadas en nuestra realidad nacional. Ambos pacientes autorizaron el reporte de los presentes casos clínicos.

\section{Caso 1}

Mujer de 49 años, con antecedentes de leucemia mieloide crónica (LMC) diagnosticada a los 39 años, desde entonces en tratamiento con imatinib y cirugía de manga gástrica a los 46 años, usuaria de fierro oral. En control por hematología se pesquisó historia de decaimiento e insomnio. $\mathrm{Al}$ examen físico, presentaba signos inespecíficos de déficit de fierro, como uñas y pelo frágil. $\mathrm{Al}$ laboratorio destacaba anemia leve, con función tiroidea, vitamina D, calcio, fósforo y PTH normales. Se indicó fierro carboximaltosa endovenoso $1.000 \mathrm{mg}$ por una vez. Tres semanas luego de la administración, evolucionó con debilidad muscular, parestesias ocasionales e hipofosfatemia persistente hasta $0,7 \mathrm{mg} / \mathrm{dL}$, por lo que fue derivada a endocrinología.

En control con endocrinología se describe asintomática. Negaba antecedentes personales o familiares de fracturas o patología ósea. Traía exámenes en donde destacaba calcio $8,3 \mathrm{mg} / \mathrm{dL}$ y fósforo $1,1 \mathrm{mg} / \mathrm{dL}$, y una reabsorción tubular de fosfato (TRP) disminuida (75,7\%) (Tabla 1$)$.

En contexto de hipofosfatemia de inicio reciente secundario a excreción renal aumentada de fósforo, y con el antecedente de exposición a fierro carboximaltosa, se diagnosticó hipofosfatemia por pérdidas renales secundaria al uso de este. A modo de confirmación, se solicitó medición de niveles de FGF23 intacto en muestra plasmática mediante técnica de ELISA (Quidel-Immutopics ${ }^{\circledR}$, Quidel Corporation. San Diego, CA, USA) que resultaron elevados en $73 \mathrm{pg} / \mathrm{ml}$. Tras cerca de 90 días de la administración, presentó normalización de la fosfatemia de forma espontánea (Figura 1).

\section{Caso 2}

Hombre de 40 años con antecedente de LMC diagnosticada a los 36 años, en tratamiento con imatinib. Sin antecedente de fracturas, talla baja, alteraciones dentales ni deformidades óseas. Presentaba valores basales de calcemia, fosfatemia y función renal normal. Posterior a los 6 meses de inicio del tratamiento con imatinib cursó con hipofosfatemia progresiva y persistente por más de 4 años, con debilidad muscular generalizada y fatiga, asociados a episodios de litiasis renal recurrente. Fue evaluado por endocrinología, destacando en los exámenes de laboratorio hipofosfatemia (1,9 mg/dL), con calcio, creatinina y niveles de vitamina $\mathrm{D}$ dentro de rango normal, y $\mathrm{PTH}$ levemente elevado. En el análisis de orina, destacaba hipercalciuria y un TRP bajo $(71,1 \%)$ (Tabla 1$)$. Se sospechó hipofosfatemia secundaria a pérdidas renales por imatinib. La medición de FGF23 intacto resultó en $30 \mathrm{pg} / \mathrm{ml}$, lo que confirmó el mecanismo independiente de FGF23. Se inició aporte oral de fosfato, asociado a hidroclorotiazida para manejo de la hipercalciuria, con mejoría clínica y normalización de la fosfatemia.

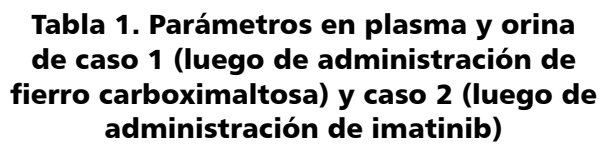

\begin{tabular}{|lccc|}
\hline & $\begin{array}{c}\text { Valor } \\
\text { normal }\end{array}$ & $\begin{array}{c}\text { Caso } \\
\text { Fósforo }(\mathrm{mg} / \mathrm{dl})\end{array}$ & $\begin{array}{c}\text { Caso } \\
\mathbf{2}\end{array}$ \\
\hline Calcio corregido (mg/dl) & $8,5-10,5$ & 1,1 & 1,9 \\
\hline Fosfatasas alcalinas (U/L) & $45-115$ & 50 & 111 \\
\hline PTH intacta (pg/mL) & $15-65$ & 124 & 74 \\
\hline 25-OH-vitamina D (ng/mL) & $>20$ & 35,5 & 31,9 \\
\hline Creatininemia (mg/dL) & $0,5-0,9$ & 0,5 & 1,1 \\
\hline Creatinina urinaria (mg/dL) & & 58 & 154 \\
\hline Fósforo urinario (mg/dL) & & 31 & 79,9 \\
\hline Fósforo urinario (mg/día) & $300-1.300$ & 465 & 770 \\
\hline TRP (\%) & $85-95 \%$ & $75,7 \%$ & $71,1 \%$ \\
\hline Calcio urinario (mg/día) & $100-300$ & 139,5 & 412 \\
\hline FGF23 intacto (pg/ml) & & 73 & 30 \\
\hline
\end{tabular}




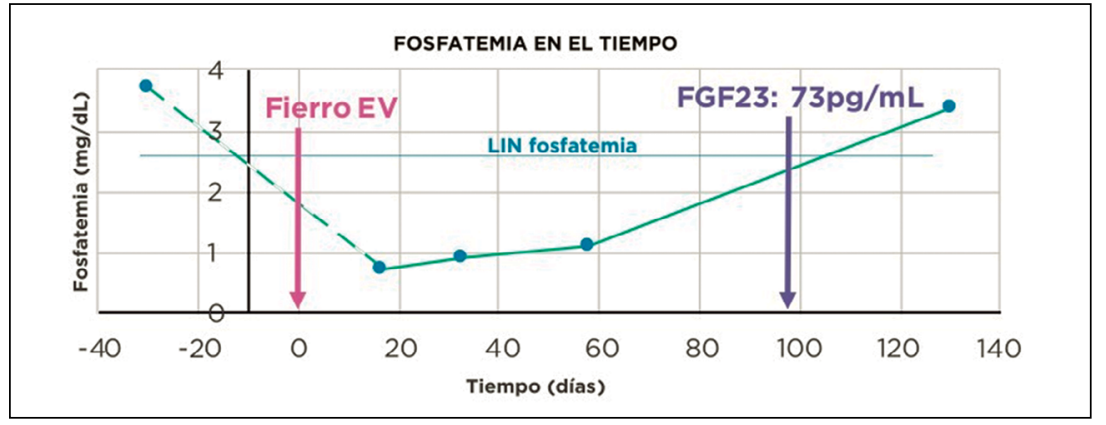

Figura 1. Caso 1. Descripción temporal de niveles plasmáticos de fósforo en relación a administración de fierro endovenoso y niveles de FGF23. LIN: Límite inferior normal.

\section{Discusión}

En este reporte presentamos dos casos de hipofosfatemia secundaria al uso de medicamentos. Tanto el uso de formulaciones de fierro carboximaltosa como de imatinib han sido descritos en la literatura internacional como causantes de hipofosfatemia ${ }^{4-7}$, sin embargo, a nuestro conocimiento, esta es la primera descripción de casos manifestados en Chile.

El FGF23 es el principal regulador de la concentración sérica de fósforo. En condiciones normales, al aumentar el fósforo sérico, aumenta FGF23, el cual reduce la reabsorción tubular de fósforo y, por consiguiente, aumenta su excreción renal $^{8}$.

El déficit de fierro se asociaría a un aumento de la expresión génica de FGF23, sin embargo, esto no se traduce en hipofosfatemia, dada la inactivación proteolítica de este factor de crecimiento. Se ha descrito que tras la administración de fierro endovenoso se normalizan los niveles de FGF234, sin embargo, algunos pacientes presentarían un aumento de FGF23 intacto determinando un incremento en la excreción de fósforo 9 . Además, secundario a los niveles elevados de FGF23 intacto disminuyen los niveles de calcitriol, provocando una hipocalcemia leve y aumento de PTH, lo cual perpetúa la hiperfosfaturia y la hipofosfatemia. Este fenómeno ocurriría con mayor riesgo con el uso de fierro en forma de carboximaltosa ${ }^{5}$. Se ha descrito un mayor riesgo en mujeres con anemia ferropénica secundaria a metrorragias y un menor riesgo en pacientes con enfermedad renal aguda o crónica ${ }^{10,11}$.

La sospecha de hipofosfatemia inducida por fierro debe plantearse frente a un paciente con hipofosfatemia tras recibir una carga endovenosa de fierro carboximaltosa, luego de haber descartado otras causas clínicamente evidentes de hipofosfatemia ${ }^{3}$ (Figura 2). En este escenario, es recomendable medir calcio, vitamina D y PTH. Luego de descartar un hiperparatiroidismo, se debe medir la excreción urinaria de fósforo a través del cálculo del TRP. De encontrarse disminuida $(<85 \%-95 \%)$, se confirma que la hipofosfatemia es inducida por pérdidas renales de fosfato. Posteriormente, de estar disponible, se debe considerar la medición de FGF23, lo que nos permitirá definir si las pérdidas están mediadas por FGF23 (valores normales-altos) o por una falla tubular renal primaria (valores bajos). El punto de corte de FGF23 que permite discriminar entre ambos grupos es un campo de activa investigación y actualmente su valor más aceptado para pacientes con hipofosfatemia es de $30 \mathrm{pg} / \mathrm{ml}^{12}$. En caso de no ser posible medir FGF23, se debe descartar disfunción tubular renal global con la medición de otros solutos en orina como glucosuria, aminoaciduria u otros.

No existen consensos en recomendaciones de control de fosfemia luego de cargas endovenosas de fierro carboximaltosa, sin embargo, pareciera ser razonable su control a las 6 a 8 semanas, tiempo habitual de control de laboratorio para ver la respuesta hematológica. Una mayor sospecha debiera surgir en caso de síntomas. Los pacientes que reciben múltiples cargas de fierro endovenoso presentan riesgo de desarrollar complicaciones crónicas de la hipofosfatemia, particularmente osteomalacia, caracterizado por dolores óseos, fracturas y pseudofracturas ${ }^{13,14}$.

El manejo consiste en la suplementación de fósforo en toda hipofosfatemia grave, mientras que en hipofosfatemias no graves dependerá de la temporalidad y sintomatología. En general, se 


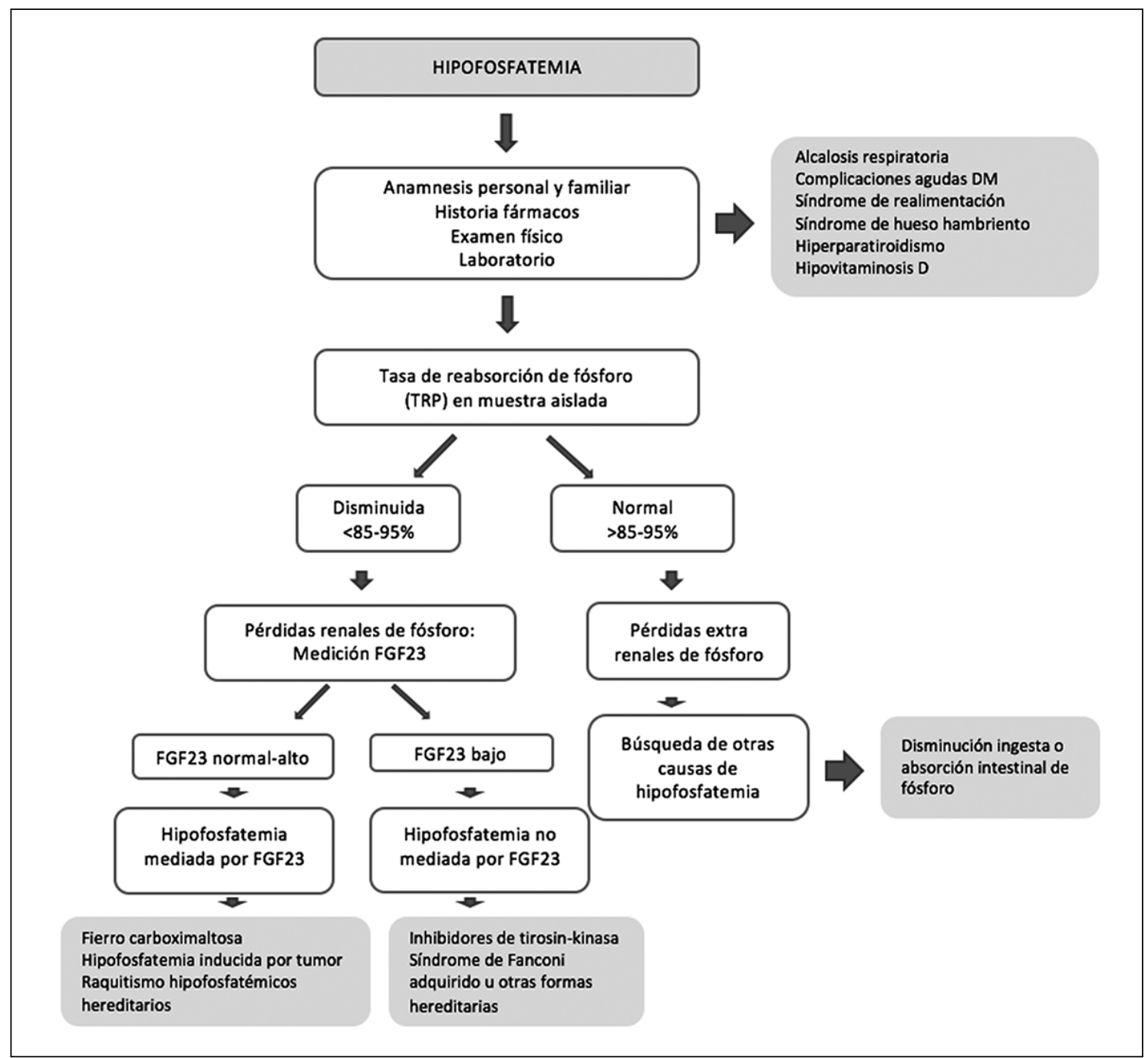

Figura 2. Enfrentamiento clínico frente a la sospecha de hipofosfatemia inducida por drogas.

sugieren aportes de 20 a $40 \mathrm{mg} / \mathrm{kg}$, dividido en 3 a 4 dosis, en forma de sales de fosfato. Actualmente en Chile no hay presentaciones comercialmente disponibles, por lo que se debe solicitar su preparación en recetario magistral. Sus principales efectos adversos corresponden a malestares gastrointestinales e hiperparatiroidismo secundario. Se debe asociar aporte de calcitriol para optimizar la absorción intestinal de fósforo ${ }^{1}$.

Por otro lado, se ha descrito la hipofosfatemia secundaria al uso de quimioterapia, en particular, inhibidores de la tirosin-kinasa como el imatinib, nilotinib y bosutinib ${ }^{6,8,15}$.
Estudios han observado una prevalencia de hasta $40 \%$ en pacientes usuarios de imatinib 6 . El mecanismo fisiopatológico permanece desconocido, pero estaría relacionado con la inhibición de la reabsorción tubular renal de fósforo. Estos mecanismos no serían mediados por FGF23 y podrían asociarse, además, a la presencia de otros elementos de falla tubular proximal parcial, como hipercalciuria, glucosuria o aminoaciduria ${ }^{6,16}$. En cuanto al metabolismo óseo, podría aumentar la maduración de osteoblastos e inhibición de osteoclastos, estimulando la formación ósea y restringiendo su resorción ${ }^{17}$. Esto se han asociado 
a calcemia normal a baja, hiperparatiroidismo secundario, aumento de excreción de fósforo e hipofosfatemia ${ }^{18,19}$.

En relación al seguimiento en pacientes usuarios de imatinib, se sugiere el control de niveles de fosfatemia y evaluación con densidad ósea (DXA), dado que aún se desconocen sus efectos a largo plazo ${ }^{8,15}$. No existe un consenso en la medición rutinaria ni temporalidad de los controles. Se sugiere la suplementación de fósforo oral dependiendo de la gravedad y síntomas asociados ${ }^{1}$. Considerando que el imatinib representa muchas veces el tratamiento de primera línea en una LMC, se recomienda el seguimiento y la suplementación de fósforo por sobre la suspensión de la droga.

En conclusión, el uso de fierro carboximaltosa e imatinib se puede asociar a hipofosfatemia, en algunos casos sintomática o grave, representando un efecto adverso poco conocido en drogas que son ampliamente utilizadas. El reconocimiento de estas asociaciones permite identificar y tratar de forma oportuna la hipofosfatemia inducida por drogas y así evitar las complicaciones asociadas a esta entidad.

\section{Referencias}

1. Florenzano P, González G. Trastornos del metabolismo del calcio y fósforo. En: Arteaga E, Baudrand R, Domínguez JM, Editores, Endocrinología clínica, segunda edición. Santiago, Chile: Editorial Mediterráneo Ltda.; 2018. p. 185-94.

2. Gaasbeek A, Meinders AE. Hypophosphatemia: an update on its etiology and treatment. Am J Med 2005; 118: 1094.

3. Florenzano P, Cipriani C, Roszko KL, Fukumoto S, Collins MT, Minisola S, et al. Approach to patients with hypophosphataemia. Lancet Diabetes Endocrinol 2020; 8: 163-74.

4. Fernández-Fernández FJ, Martín-Fernández A. Parenteral iron as a cause of hypophosphataemia. BMJ 2014; 349: g4616.

5. Schaefer B, Würtinger $P$, Finkenstedt A, Braithwaite V, Viveiros A, Effenber M, et al. Choice of High-Dose Intravenous Iron Preparation Determines Hypophosphatemia Risk. PloS One 2016; 11 (12): e0167146.

6. Osorio S, Noblejas AG, Durán A, Steegmann JL. Imatinib mesylate induces hypophosphatemia in patients with chronic myeloid leukemia in late chronic phase, and this effect is associated with response. Am J Hematol 2007; 82: 394.
7. Berman E, Girotra M, Cheng C, Chanel S, Maki R, Shelat $\mathrm{M}$, et al. Effect of long term imatinib on bone in adults with chronic myelogenous leukemia and gastrointestinal stromal tumors. Leuk Res 2013; 37: 790.

8. Sneddon WB, Ruiz GW, Gallo LI, Xiao K, Zhang Q, Rbaibi Y, et al. Convergent Signaling Pathways Regulate Parathyroid Hormone and Fibroblast Growth Factor-23 Action on NPT2A-mediated Phosphate Transport. J Biol Chem 2016; 291: 18632.

9. Schouten BJ, Hunt PJ, Livesey JH, Frampton CM, Soule SG. FGF23 elevation and hypophosphatemia after intravenous iron polymaltose: a prospective study. J Clin Endocrinol Metab 2009; 94: 2332.

10. Wolf M, Koch TA, Bregman DB. Effects of iron deficiency anemia and its treatment on fibroblast growth factor 23 and phosphate homeostasis in women. J Bone Miner Res 2013; 28: 1793.

11. Zoller H, Schaefer B, Glodny B. Iron-induced hypophosphatemia: an emerging complication. Curr Opin Nephrol Hypertens. 2017; 26 (4): 266-75.

12. Endo I, Fukumoto S, Ozono K, Namba N, Tanaka H, Inoue $\mathrm{D}$ et al. Clinical usefulness of measurement of fibroblast growth factor 23 (FGF23) in hypophosphatemic patients Proposal of diagnostic criteria using FGF23 measurement. Bone 42 (2008) 1235-9.

13. Wolf M, Chertow GM, Macdougall IC, Kaper R, Krop J, Strauss W. Randomized trial of intravenous iron-induced hypophosphatemia. JCI Insight. 2018; 3 (23) Epub 2018 Dec 6.

14. Anand G, Schmid C. Severe hypophosphataemia after intravenous iron administration. BMJ Case Rep. 2017; 2017 Epub 2017 Mar 13.

15. Owen S, Hatfield A, Letvak L. Imatinib and altered bone and mineral metabolism. N Engl J Med 2006; 355: 627; author reply 628 .

16. Francoise H, Coppo P, Hayman JP, Fouqueray B, Mougenot B, Ronco P. Partial Fanconi Syndrome Induced by Imatinib Therapy: A Novel Cause of Urinary Phosphate Loss. Am J Kidney Dis. 2008; 51 (2): 298-301.

17. Dewar AL, Farrugia AN, Condina MR, Bik To L, Huges TP, Vernon-Roberts B, et al. Imatinib as a potential antiresorptive therapy for bone disease. Blood 2006; 107: 4334-7.

17. Berman E, Nicolaides M, Maki RG, Fleisher M, Chanel S, Scheu K, et al. Altered Bone and Mineral Metabolism in Patients Receiving Imatinib Mesylate. N Engl J Med 2006; 354: 2006-13.

19. Grey A, O'Sullivan S, Reid IR, Browett P. Imatinib mesylate, increased bone formation, and secondary hyperparathyroidism. N Engl J Med 2006; 355: 2494. 